Proceedings of the International Conference on Instrumentation for High Energy Physics,

Dubna, USSR, Sept. 8-12, 1970
UCRL-20135

Preprint

Conf-700902-..7

PARTICLE DETECTORS BASED ON NOBLE LIQUIDS

Richard A. Muller, Stephen E. Derenzo, Robert G. Smits Haim Zaklad, and Luis W. Alvarez

September 1970

AEC Contract No. W-7405-eng-48
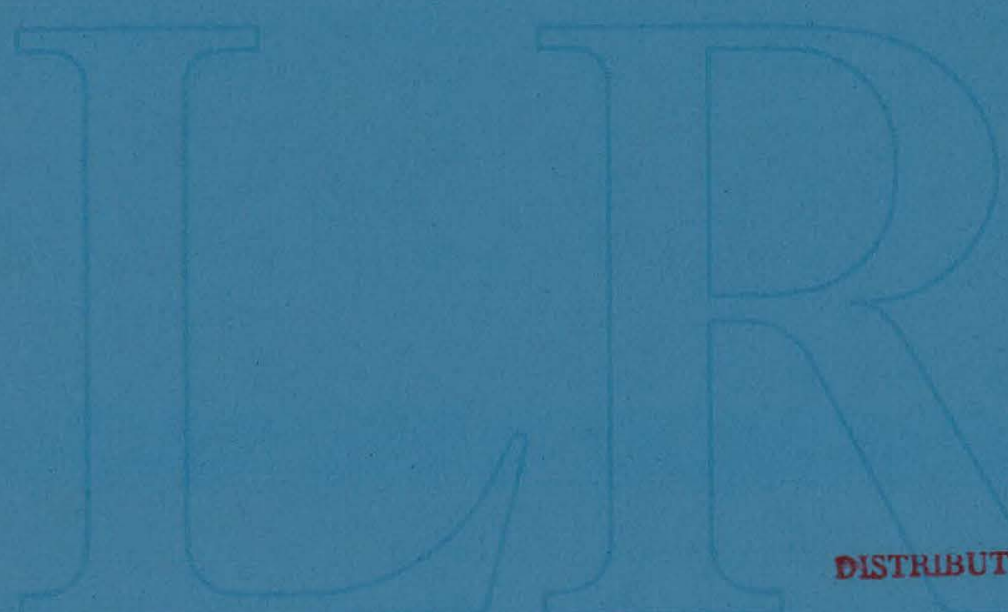

DISTRUBUTION OF THUS DOCUMENT IS UNLIMITED 


\section{DISCLAIMER}

This report was prepared as an account of work sponsored by an agency of the United States Government. Neither the United States Government nor any agency Thereof, nor any of their employees, makes any warranty, express or implied, or assumes any legal liability or responsibility for the accuracy, completeness, or usefulness of any information, apparatus, product, or process disclosed, or represents that its use would not infringe privately owned rights. Reference herein to any specific commercial product, process, or service by trade name, trademark, manufacturer, or otherwise does not necessarily constitute or imply its endorsement, recommendation, or favoring by the United States Government or any agency thereof. The views and opinions of authors expressed herein do not necessarily state or reflect those of the United States Government or any agency thereof. 


\section{DISCLAIMER}

Portions of this document may be illegible in electronic image products. Images are produced from the best available original document. 


\title{
PARTICLE DETECTORS BASED ON NOBLE LIQUIDS
}

\author{
Richard A. Muller \\ University of California Space Sciences Laboratory \\ Berkeley, California \\ and \\ Stephen E. Derenzo, Robert G. Smits, \\ Haim Zaklad, and Luis $W$. Alvarez \\ Lawrence Radiation Laboratory, University of California \\ Berkeley, California
}

September 1970

\begin{abstract}
In order to build a thin particle detector with 10 micron spatial resolution and automatic readout, the avalanche of ionization electrons in high electric fields in liquid argon and liquid xenon has been studied. We present a scheme using an array of points that could be used to make a reliable liquid argon filled detector. The avalanche pulses in liquid xenon have a rise time more than three orders of magnitude faster than that in liquid argon, suggesting that the positive charge carriers are holes, and making possible a detector with a time resolution of better than 100 nanoseconds. A direct observation of hole conduction is described.
\end{abstract}

\footnotetext{
This report was prepared as an account of work sponsored by the United States Government. Neither the United States nor the United States Atomic Energy Commission, nor any of their emplnye.es, nor any of their contractors, subcontractors, or their employees, makes any warranty, express or implied, or assumes any legal liability or responsibility for the accuracy, completeness or usefulness of any information, apparatus, product or process disclosed, or represents that its use would not infringe privately owned rights.
} 
We have been studying the avalanche of ionization electrons in high electric fields in the noble liquids, argon and xenon. We have observed the avalanche in both of these liquids 1,2 at fields of about $10^{6}$ volts $/ \mathrm{cm}$. We believe the existence of this avalanche makes possible a particle detector with a thickness of $100 \mathrm{microns}$ or less, with a spatial resolution of \pm 10 microns. Such a detector would introduce $<0.005$ radiation lengths. Four our studies, we have constructed detectors using single wires 2.5 to 20 microns in diameter, filled with purified ${ }^{1,2}$ liquid argon and xenon in a geiger counter geometry.

We have been unable to make a reliable detector using liquid argon. The efficiency for particle detection has never been better than about $20 \%$. The low efficiency is due to the existence of "hot spots" on the wire, smaller than $4 \mathrm{~mm}$ in length. The wire has approximately $100 \%$ efficiency for avalanche in the region of these hot spots, and almost zero efficiency elsewhere. The hot spots may be due to sub-microscopic ir regularities that locally increase the field strength. We have been unable to control the number of hot spots that occur on a wire submerged in liquid argon. If we attempt to improve the efficiency by increasing the voltage, we observe spontaneous sparkling independent of the presence of ionizing radiation. However we have observed that a hot spot always occurs on the tip of an etched tungsten point, if the tip radius is one micron or less. It should therefore be possible to construct a useful liquid argon particle 
detector by using an array of sharp points. Arrays of such points have been made with 25 micron spacing by Done Cone and his group at the Stanford Research Institute using a vapor deposition technique. A typical array is shown in figures 1 and 2 .

Our efforts with liquid xenon have been much more successful. With a 3.5 micron diameter tungsten wire, submerged in purified liquid xenon, ${ }^{3}$ typical operation is as follows. At low voltages, below about -500 volts (voltage of a concentric cylindrical cathode about 4 $\mathrm{mm}$ in radius with respect to the wire) we observe only ionization charges collected on the wire. Between $-500 \mathrm{~V}$ and $-3.5 \mathrm{KV}$ the ionization electrons avalanche in the vicinity of the wire, giving a gain of up to $10^{4}$. But unlike our results with argon the efficiency for avalanche is good all along the wire, somewhere between $25 \%$ and $100 \%$. At higher voltages we have observed a sparking mode, with the chamber operating as a D-C spark Chamber. However the efficiency of the chamber in this mode is very low, probably less than $1 \%$. The spark does not seem to damage the fine wire. A hundred volts or so above the D-C spark chamber voltage the sparks occur spontaneously, in the absence of ionizing radiation. Because of the larger pulses in the spark mode, we are attempting to improve the efficiency of our detector by pulsing it to higher voltages. We know that our chambers can be pulsed to greater than $10 \mathrm{KV}$ for $1 \mu \mathrm{s}$ without spontaneous sparking, even when the D-C breakdown voltage is only $5 \mathrm{KV}$. 


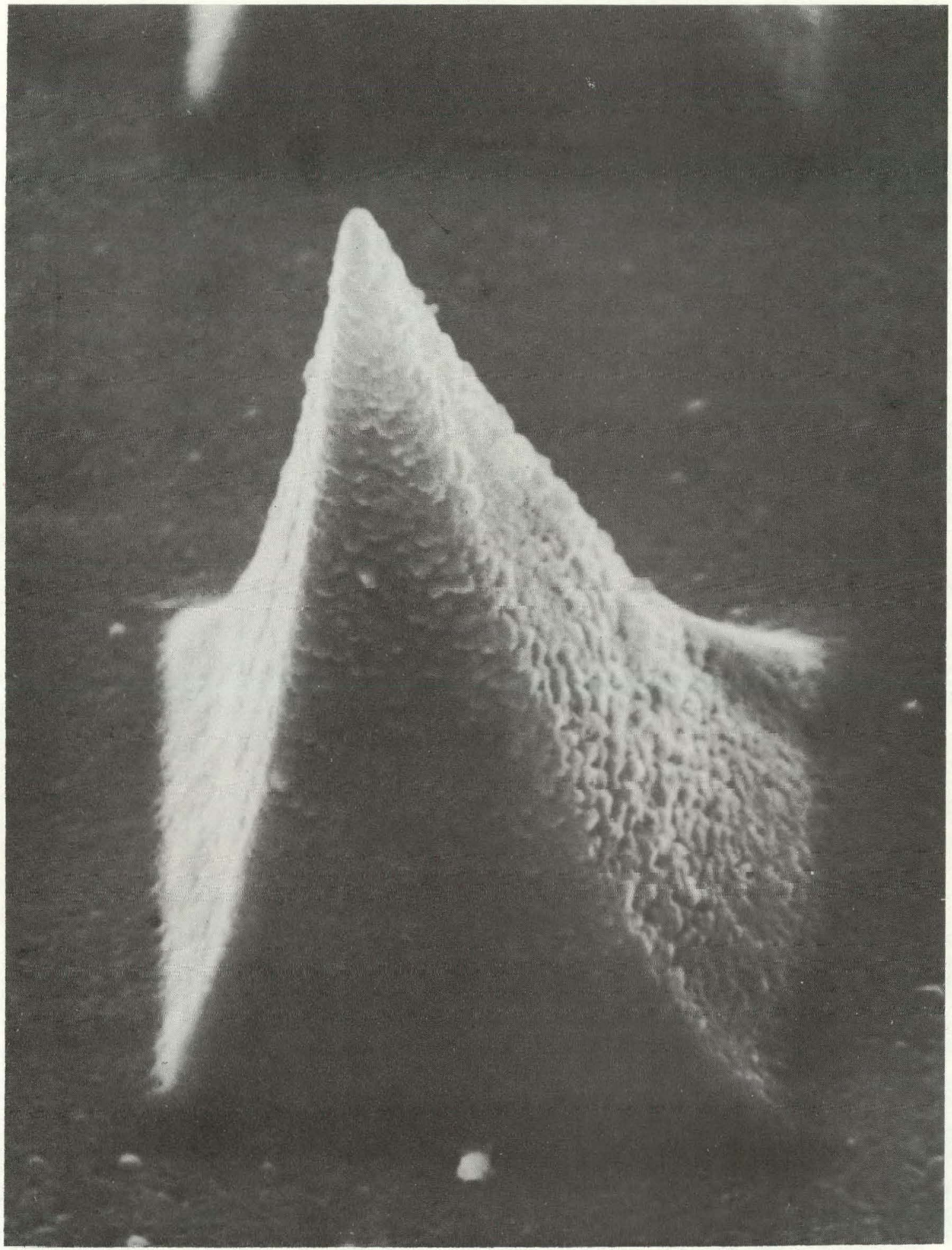

XBB 706-2549

Fig. 1. A pyramid made by Don Cone and his group at Stanford Research Institute. (Scanning electron microscope photograph.) The height of the pyramid is about 15 microns, and the material is an alumina-tungsten alloy. 


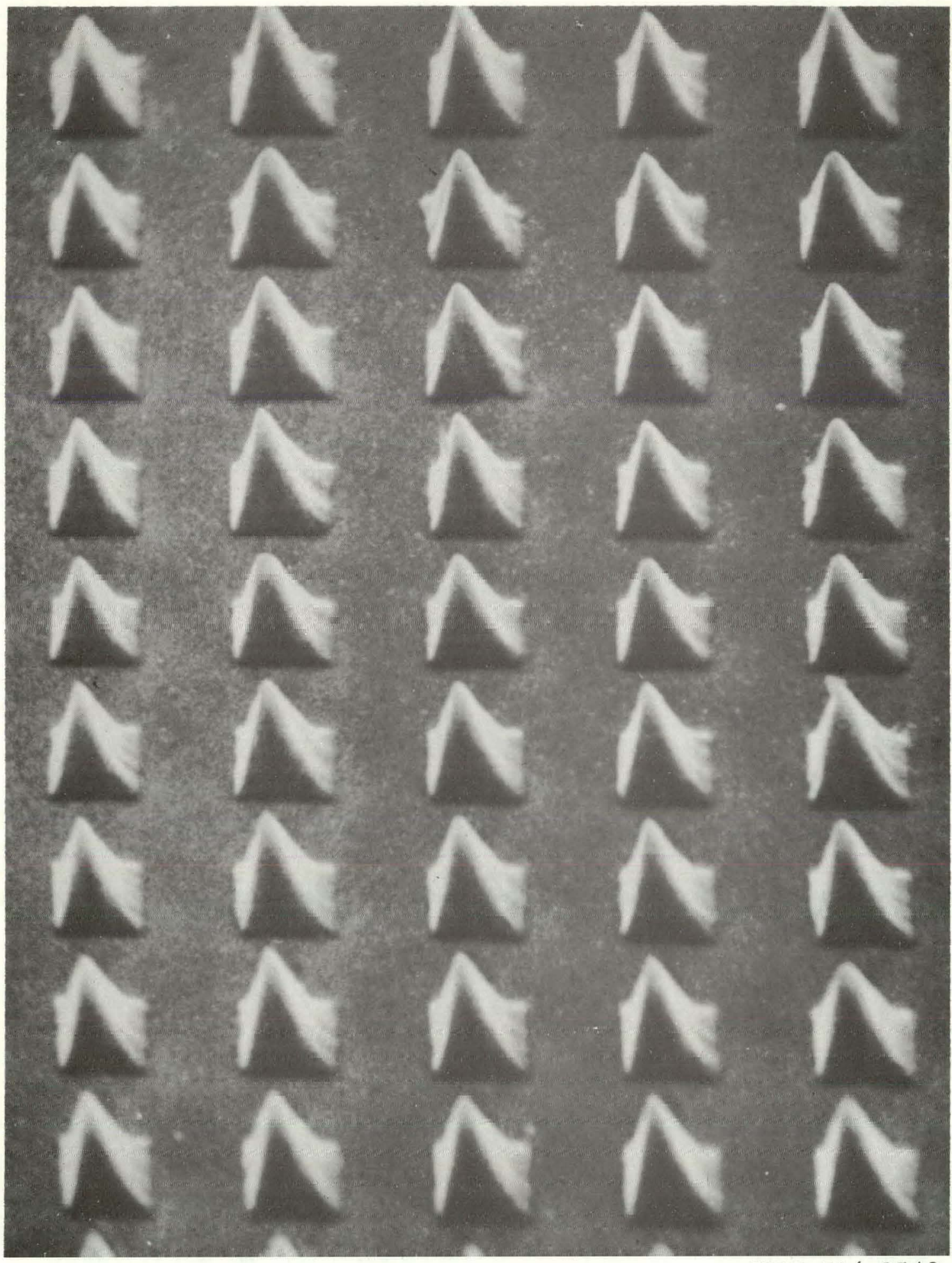

XBB $706-2548$

Fig. 2. An array of pyramids. The spacing between tips is 25 microns. 
The avalanche pulses in liquid xenon have one extremely interesting feature. Whether the avalanche occurs on a fine wire or an etched tungsten point, the rise-time is very fast, less than 6 nanoseconds, our measurement limited by the rise-time of our oscilloscope. For argon the rise-time is more than three orders of magnitude longer. The rise-time in our counters is determined by the motion of positive charges away from the wire, since most of the avalanche is created in the vicinity of the wire and the electrons move only a short distance to the anode. The fast rise time therefore indicates high positive charge mobility, suggesting that the positive charge carriers are holes.

We have done one other experiment that suggests that hole conduction occurs in purified liquid xenon under the influence of high electric fields. We constructed a chamber with two spherical electrodes $3 / 16$ inches in diameter, spaced 0.05 inches apart. On the lower sphere we placed a small $\mathrm{Am}^{241}$ alpha emitter. The range of the $5.5 \mathrm{MeV}$ alphas is about 0.002 inches in both liquid argon and 11quid xenon. Therefore the ionization occurs in the vicinity of the lower electrode. If the lower electrode is at negative voltage we observe the motion of the negative charges towards the upper electrode, and if the lower electrode is positive we observe the motion of positive charges.. At $15 \mathrm{KV}$, in liquid argon, the drift time for the negative charges is less than 0.5 microseconds, and the drift time for the positive charges is greater than 50 microseconds, consistent with 
previous observations $4,5,6$ that the negative carries are electrons and the positive carriers are ions. Using the same chamber filled with liquid xenon we find that the drift time for both positive and negative charges is 1 ess than 0.5 microseconds, indicating both electron and hole conduction.

The pulse height when the positive charge motion was observed was only $30 \%-50 \%$ that for the electron motion, perhaps indicating that holes are more easily trapped on the impurities in our xenon than are electrons.

The fast rise time of the pulses in liquid xenon has important consequences for high energy physics experiments. It should allow the design of a detector with good time resolution (20-40 nanoseconds) as well as good spatial resolution, using the conventent scanned readout described in references 7 and 8 .

For the details of some of the experiments described in this paper we refer the reader to reference 2 .

We would like to thank. Dennis B. Smith and Carl Pennypacker for help in all aspects of this work, and we are grateful to Charles Kittel for a very informative conversation. We are indebted to Buck Buckingham, Ernie Currier, Joe Savignano, and Tony Vuletich for help in the construction and maintenance of our apparatus. 
FOOTNOTES AND REFERENCES

1. Prospect of High Spatial Resolution for Counter Experiments: A New Particle Detector Using Electron Multiplication in Liquid Argon. Derenzo, Muller, Smits, Alvarez, UCRL-19254. Published in 1969 NAL Summer Study Reports, Aspen, Colorado.

2. Recent Developments in High Resolution Noble Liquid Counters. Derenzo, Smits, Zaklad, Alvarez, and Muller. UCR L-20118. To be published in the 1970 NAL Summer Study Report.

3. We have purified our xenon using a purifier similar to that used for argon, described in reference 2 . We have not measured the amount of residual impurities, although we know that when purifying argon the amount of residual oxygen is less than 200 parts per million.

4. G. W. Hutchinson, Nature 162, 610 (1948); thesis, St. Johns College, Cambridge, 1951 (unpublished).

5. Davidson and Larsh, Phys. Rev., 74, 220 (1948); 77, 706 (1950).

6. John Marshall, Rev. Sci. Inst. 25, 232 (1954).

7. In principle, a liquid argon detector also has good time resolution because although the rise-time is determined by the mobility of positive charges, the delay between the passage of an ionizing particle and rise of a pulse to a certain height is deter: mined by the electron velocity. Thus although the pulse is slow, 
it reaches a certain pulse height at a well determined time.

8. For a detailed discussion of the readout methods applicable to the high resolution chambers discussed here, see Integrated Circuit Readout for Closely Spaced Wire Array8, Haim Zaklad, NAL 1970 Summer Study Report SS-181, also UCR L-20123. 


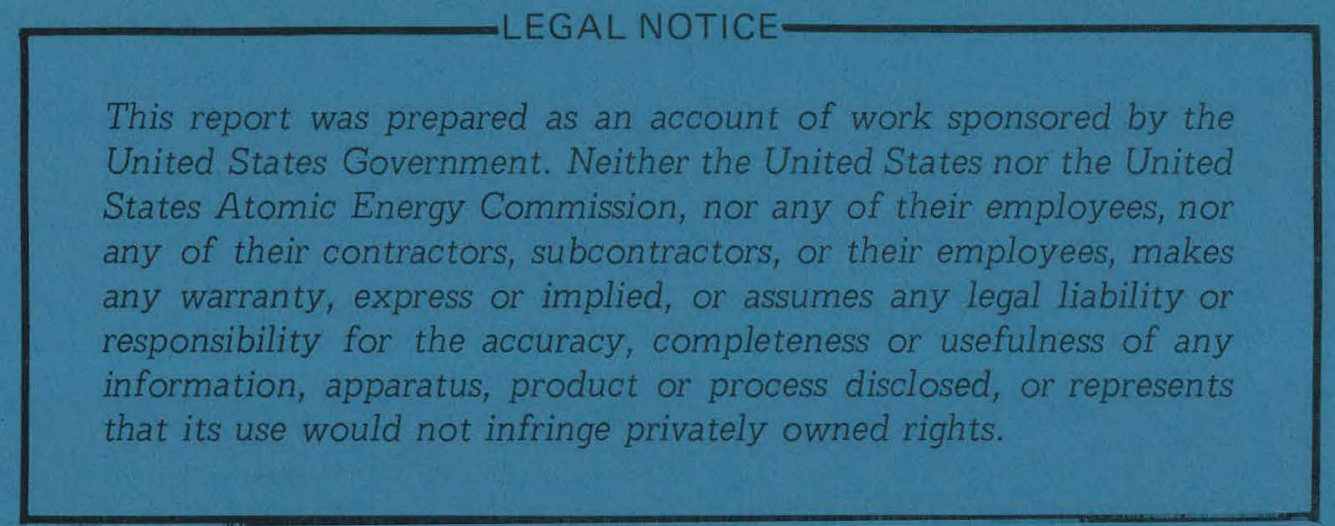


